\title{
Rôle de la B-lactoglobuline dans l'activité proliférative du lactosérum
}

\author{
F Moulti-Mati 1, A Mati 2, J Capiaumont 1, F Belleville 1, \\ G Linden 2, P Nabet 1
}

\author{
1 Université de Nancy l, faculté de médecine, laboratoire de biochimie, \\ BP 184, 54505 Vandoeuvre Cedex, et Institut des biotechnologies de Nancy; \\ 2 Université de Nancy I, faculté des sciences, laboratoire de biochimie appliquée associé à I'INRA, \\ BP 239, 54506 Vandœuvre Cedex, France
}

(Reçu le 5 novembre 1990; accepté le 17 juin 1991)

Résumé - Le lactosérum est capable de remplacer le sérum de veau fœtal (SVF) dans des cultures d'hybridomes à court terme (48 h). La B-lactoglobuline variant $\mathrm{A}$ ou $\mathrm{A} / \mathrm{B}$ a la même activité mitogénique que le lactosérum. L'activité du variant $B$ est significativement plus faible. Le maximum d'activité est obtenu pour des concentrations de $3 \mathrm{~g} / \mathrm{l}$ variant $A$ et $A / B$ et de $0,75 \mathrm{~g} / \mathrm{l}$ variant $\mathrm{B}$. Pour la croissance cellulaire à plus long terme, $1 \%$ de SVF doit être ajouté au lactosérum. La B-lactoglobuline variant $A / B(2,7 \mathrm{~g} /)$ supplémenté par $1 \%$ de SVF a le même effet mitogénique que le lactosérum.

lactosérum / B-lactoglobuline / variant B-lactoglobuline / activité mitogénique / culture cellulaire

Summary - B-lactoglobulin part in mitogenic activity of bovine whey. Bovine whey is able to replace fetal calf serum (FCS) in short-term hybridoma culture (48 h). B-lactoglobulin (major protein of bovine whey) with a level of $\approx 3 \mathrm{~g} / \mathrm{l}$ variant $A$ or $A / B$ has the same effect as that of whey on DNA synthesis. The activity of variant $B$ is significantly lower. The optimal mitogenic activity of variant $A$, $A / B$ and $B$ is obtained at concentrations of $3 \mathrm{~g} /, 3 \mathrm{~g} / \mathrm{l}$ and $0.75 \mathrm{~g} / \mathrm{l}$ respectively. For monitoring cellular proliferation in long-term culture, the whey must be supplemented with $1 \%$ FCS. B-lactoglobulin variant $A / B$ at $2.7 \mathrm{~g} / 1$ (with 1\% FCS) also promotes long-term proliferation and can replace whey. Blactoglobulin or FCS (1\%) used independently does not ensure cell survival.

whey / B-lactoglobulin / B-lactoglobulin variant / mitogen effect / cell culture 


\section{INTRODUCTION}

Le lait humain ou bovin a été quelquefois utilisé en remplacement de sérums d'animaux pour supplémenter les milieux de culture (Klagsbrung, 1978; Sereni et Baserga, 1981). Dans des travaux précédents, nous avons montré que du lactosérum bovin pouvait remplacer le sérum de veau fœtal (SVF) dans la culture d'hybridomes à court terme $(48 \mathrm{~h})$. Pour des cultures à plus long terme, il faut ajouter au lactosérum 1\% de SVF (Nabet et al, 1985; Linden et al, 1988; Damerdji et al, 1988; Derouiche et al, 1990). Cependant, le lactosérum, comme le SVF, est un milieu complexe; afin d'obtenir un milieu de culture de composition mieux définie, nous avons tenté de remplacer le lactosérum par une protéine purifiée du lait. Nous avons choisi la B-lactoglobuline (B-Lg) qui est la protéine la plus abondante du lactosérum (environ $3 \mathrm{~g} / \mathrm{l}$ ). Elle représente environ $50 \%$ des protéines totales du lactosérum. Par ailleurs, ses caractéristiques physico-chimiques font d'elle un bon transporteur de substances hydrophobes, propriété intéressante en culture cellulaire.

La B-Lg est une chaîne polypeptidique de 162 acides aminés. On connaît 7 variants génétiques ( $A, B, C, D, E, F$ et $G$ ); seuls les variants $A$ et $B$ sont fréquemment rencontrés (Eigel et al, 1984). Ce polymorphisme a sans doute une signification biologique, par exemple, l'action inhibitrice sur la B-Lg de la phosphatase de la rate est fonction du variant; ainsi le pourcentage d'inhibition est de $40 \%$ pour le variant $A, 22 \%$ pour le variant $B$ et $12 \%$ pour le variant $\mathrm{C}$ (Farrell et Thomson, cités par Alais, 1984).

In vivo, son rôle biologique demeure inconnu; des travaux in vitro montrent que la B-Lg est capable de se lier à des substances hydrophobes : lipides (Brown, 1984; Diaz de Villegas et al, 1987), acides gras (Polet-Spieker et Polet, 1981) et rétinol (Rask et al, 1979; Fugate et Song, 1980; Godovac-Zimmermann et al, 1985). D'ailleurs, la B-Lg présente des homologies de structure avec la Retinol binding protein; le résidu Trp 19 serait le site de fixation (Papiz et al, 1986; GodovacZimmermann et Braunitzer, 1987; Godovac-Zimmermann, 1988). Tout récemment, Said et al (1989) ont montré chez le rat que le rétinol pouvait être absorbé à travers l'intestin en se liant à la BLg bovine.

Dans le présent travail, nous avons étudié l'action mitogénique de différents variants de la $B-\mathrm{Lg}$ sur des cultures d'hybridomes. Parallèlement à la prolifération des cellules, nous avons testé la sécrétion des anticorps monoclonaux.

\section{MATÉRIEL ET MÉTHODES}

\section{Préparation des protéines du lait (Société ASMAR, Corcieux, France)}

Le lactosérum obtenu industriellement est concentré ( $6 \%$ en protéines), écrémé et filtré, puis il est porté quelques secondes à $84^{\circ} \mathrm{C}$ et ultrafiltré à $50{ }^{\circ} \mathrm{C}$ avec une membrane à seuil de coupure $10000 \mathrm{Da}$ ( $10,5 \%$ en protéines). La solution de protéines est pasteurisée, concentrée par évoporation sous vide ( $55 \%$ en protéines), puis séchée en tour par la chaleur. Ces protéines sont appelées protéines du lait ou lactosérum lorsqu'elles sont remises en solution. Nous les utilisons généralement à la concentration de $20 \mathrm{~g} / \mathrm{l}$.

\section{Purification de la B-lactoglobuline}

La méthode utilisée est celle décrite par Lung et al (1987). Dans ce protocole, la B-Lg est isolée à partir du lactosérum par chromatographie d'échange d'ions sur DEAE Sephacel (Pharmacia, Suède). Le lactosérum est dialysé contre un 
tampon Tris- $\mathrm{HCl} 0,1 \mathrm{~mol} / \mathrm{l}, \mathrm{pH} 7,5,0,02 \mathrm{~mol} / 1$ $\mathrm{NaCl}$; le même tampon est utilisé pour équilibrer le DEAE Sephacel. Le mélange est filtré sous vide. Des tampons, de force ionique plus élevée, sont ensuite ajoutés successivement $(0,04$ $\mathrm{mol} / /$ et $0,25 \mathrm{~mol} / \mathrm{l}$ en $\mathrm{NaCl}$ ). La $\beta-\mathrm{Lg}$ est récupérée dans le dernier filtrat. Cette fraction est ensuite purifiée par chromatographie de filtration sur gel de Sephadex $\mathrm{G} 100$ (colonne $5 \times 100$ $\mathrm{cm}$ ); un tampon phosphate de sodium $0,02 \mathrm{~mol} /$ I, $\mathrm{pH} 6,7, \mathrm{NaCl} 0,1 \mathrm{~mol} / /$ est utilisé pour l'élution.

La pureté de la $B$ - Lg est vérifiée par électrophorèse PAGE en milieu non dissociant, en utilisant la méthode d'Hillier (1976) modifiée : électrophorèse verticale avec un gel $(T: 9 \%, C$ : $2,7 \%$ ) de $1,5 \mathrm{~mm}$ d'épaisseur, tampon de migration Tris $0,05 \mathrm{~mol} / /$, glycine $0,07 \mathrm{~mol} / \mathrm{l}, \mathrm{pH} 8,3$. La pureté est également vérifiée par chromatographie FPLC sur une colonne échangeuse d'anions (Mono Q).

Les variants $A$ et $B$ de la $B-L g$ ont été isolés à partir de lait de vaches homozygotes alors que le variant $A / B$ provient d'un mélange de laits.

\section{Culture de cellules}

Deux souches d'hybridomes ont été utilisées : hybridome Mark 3 , sécrétant un anticorps monoclonal $\lg _{1}$ anti-chaîne $\mathrm{K}$ des immunoglobulines de rat (don du Professeur Bazin, laboratoire d'immunologie expérimentale, faculté de médecine, Bruxelles), hybridome anti-HPL sécrétant une $\operatorname{lgG}_{1}$ anti-hormone lactogène placentaire humaine préparé dans notre laboratoire; ces cellules sont entretenues en routine dans du RPMI 1640 (Seromed) supplémenté avec 10\% de sérum de veau fœtal (Flow Laboratoires, lot 9042362 - Puteaux, France) chauffé à $56^{\circ} \mathrm{C}$ pendant $30 \mathrm{~min}$ et $4 \mathrm{mmol} / \mathrm{l}$ de glutamine.

\section{Test de croissance à court terme (mesure de la synthèse d'ADN)}

Ce test est utilisé pour sélectionner les produits stimulant la croissance cellulaire. Les produits trouvés actifs sont ensuite testés en culture à plus long terme. Les suspensions cellulaires
( $4 \times 10^{5}$ cellules $/ \mathrm{ml}$ ) sont déposées dans des microplaques de 96 puits (Falcon, Becton, Dickinson, Grenoble, France) à raison de $100 \mu \mathrm{l}$ par puits; $100 \mu \mathrm{l}$ de produits à tester, dilués dans du RPMI sont ajoutés ( 6 déterminations sont faites pour chaque produit). Les plaques sont incubées $48 \mathrm{~h}$ à $37{ }^{\circ} \mathrm{C}$ dans un incubateur à $5 \%$ de $\mathrm{CO}_{2}$ (Jouan EG $110 \mathrm{IR}$, France); $50 \mu \mathrm{l}$ de ${ }^{3} \mathrm{H}$ thymidine à $1 \mu \mathrm{Ci} / \mathrm{ml}$ (activité spécifique $4,5 \mathrm{Ci}$ / mmol, Amersham, les Ulis, France) sont ajoutés pendant les dernières $24 \mathrm{~h}$ d'incubation. Les cellules sont collectées (Harvester Titertek Skarton) lavées, la radioactivité est comptée.

\section{Test de prolifération cellulaire}

Les cultures sont réalisées sans adaptation préalable des cellules aux milieux à tester; elles sont maintenues jusqu'à stabilisation ou chute de la prolifération cellulaire. Les cellules sont réparties dans des flacons statiques de $25 \mathrm{~cm}^{2}$ à raison de $15 \times 10^{4}$ cellules $/ \mathrm{ml}$. Lorsque les cellules sont à confluence (environ tous les 2 ou 3 jours), un comptage des cellules vivantes et mortes est effectué après coloration avec une solution de bleu trypan à $0,5 \%$; puis les cellules sont réensemencées dans du milieu neuf, à la densité cellulaire de départ, c'est-à-dire $15 \times$ $10^{4}$ cellules $/ \mathrm{ml}$.

\section{Mesure de la sécrétion des immunoglobulines}

Elle se fait par test ELISA (Douillard et Hoffman, 1983), en utilisant comme premier anticorps un sérum de chèvre anti-lgG de souris et un deuxième anticorps conjugué à la peroxydase (Tago, Burlingame, Etats-Unis).

\section{Tests statistiques}

L'analyse statistique des données est traitée par le logiciel ANOVA, donnant les moyennes $(m)$, les écart types (ET), le coefficient de variation (CV) et le test $t$ de Student, pour la signification des résultats. 


\section{RÉSULTATS}

\section{Purification de la $\beta$-lactoglobuline}

La figure 1 donne le chromatogramme obtenu après filtration sur gel. La zone hachurée représente le pic de lactoglobuline qui est recueilli. La chromatographie FPLC permet de calculer un degré de pureté qui est d'environ $98 \%$ pour les variants $A / B$ et $B$. L'électrophorèse confirme que dans les fractions purifiées, seules apparaissent les bandes correspondant à la $\beta$ - Lg.
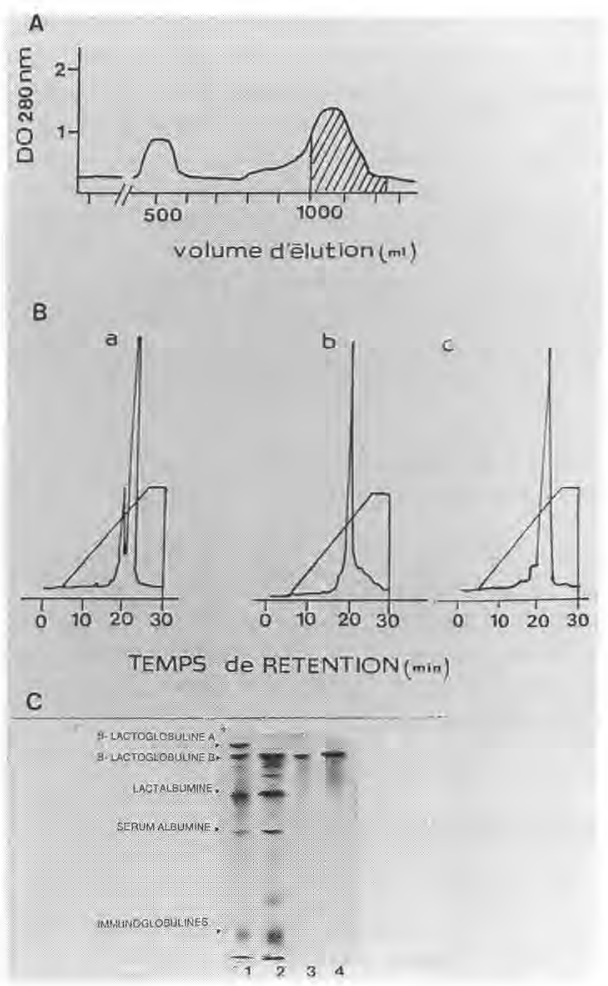

\section{Test de croissance à court terme}

Différents variants de la $\beta$ - $\mathrm{Lg}$ sont testés à différentes concentrations en présence ou non de sérum de veau fœtal à la concentration de $1 \%$. Avec chaque série d'expériences, on fait un témoin positif (cellules cultivées en présence de $10 \%$ de SVF dans les mêmes conditions que les essais).

Les résultats sont consignés dans les figures $2 \mathrm{~A}$ et $2 \mathrm{~B}$. La courbe dose-réponse est une courbe en cloche; de fortes concentrations en $\beta$ - $\mathrm{Lg}$ sont inhibitrices.

Fig 1. Purification de la B-lactoglobuline. A. Filtration sur gel (Sephadex G100) des protéines issues de la chromatographie sur DEAE Sephacel, colonne $5 \times 100 \mathrm{~cm}$, tampon d'élution phosphate de sodium 0,02 mol/l, $\mathrm{pH} 6,7, \mathrm{NaCl} 0,1$ $\mathrm{mol} / \mathrm{l}$, débit $60 \mathrm{ml} / \mathrm{h}$, dépôt $1,5 \mathrm{~g}$ de protéines. $\mathrm{B}$. Chromatographie FPLC; colonne échangeuse d'ions Mono Q. Tampon d'élution : Tris- $\mathrm{HCl} 20$ $\mathrm{mmol} / \mathrm{l}, \mathrm{pH} 7$, gradient de $\mathrm{NaCl} 0-0,35 \mathrm{~mol} / \mathrm{l}$, (a) variant $A / B$, (b) variant $B$, (c) variant $A$. Débit d'élution $1 \mathrm{ml} / \mathrm{min}$. C. Electrophorèse verticale sur PAGE, milieu non dissociant, tampon de gel : Tris- $\mathrm{HCl} 0,38 \mathrm{~mol} / \mathrm{l}, \mathrm{pH} 8,9$; tampon d'électrode : Tris $0,05 \mathrm{~mol} / \mathrm{l}$, glycine $0,07 \mathrm{~mol} / \mathrm{l}, \mathrm{pH} 8,3$; courant continu $60 \mathrm{~mA}, 500 \mathrm{~V}, 30 \mathrm{~W}$. Piste 1: lactosérum contenant le variant $A / B$ de la $B$ lactoglobuline; piste 2 : lactosérum contenant le variant $B$ de la $\beta$-lactoglobuline; pistes 3 et $4: B$ lactoglobuline variant $B$ purifié.

B-lactoglobulin purification. A. Gel filtration (Sephadex G100) of $1.5 \mathrm{~g}$ proteins from DEAE Sephacel batch chromatography, on a $5 \times 100 \mathrm{~cm}$ column, with sodium phosphate elution buffer (0.02 mol/l $\mathrm{pH} 6.7, \mathrm{NaCl} 0.1 \mathrm{~mol} / \mathrm{l})$, flow rate 60 $\mathrm{ml} / \mathrm{h}$. B. FPLC chromatography; ion exchange mono- $Q$ column; elution buffer: Tris- $\mathrm{HCl} 20$ mmolll, $\mathrm{pH} 7, \mathrm{NaCl}$ gradient $0-0.35$ molll, (a) Blactoglobulin variant $A / B$, (b) B-lactoglobulin variant $B$, (c) $B$-lactoglobulin variant $A$. Flow rate 1 $\mathrm{ml} / \mathrm{min}$. C. PAGE vertical electrophoresis in non dissociated medium; gel buffer: Tris- $\mathrm{HCl} 0.38$ molll, pH 8.9; electrode buffer: Tris 0.05 molll, glycine $0.07 \mathrm{~mol} / \mathrm{l}, \mathrm{pH}$ 8.3; constant current : $60 \mathrm{~mA}, 500 \mathrm{~V}, 30 \mathrm{~W}$. Lane 1 : whey containing B-lactoglobulin $A / B$; lane 2 : whey containing B-lactoglobulin B; lanes 3 and 4 : purified B-lactoglobulin $B$. 


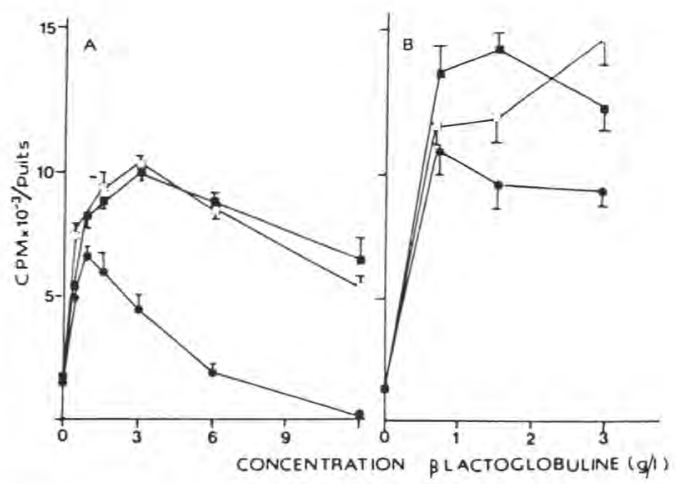

Fig 2. Test de croissance à court terme : mesure de l'incorporation de ${ }^{3} \mathrm{H}$ thymidine dans l'ADN. Les variants de la $B$-lactoglobuline ( $\square$ variant $A$, • variant $B$, variant $A / B$ ) sont testés en absence de sérum de veau fœetal (A) ou en présence de $1 \%$ de sérum de veau fœtal (SVF) (B). Chaque résultat est la moyenne de 6 déterminations.

Short-term growth test: ${ }^{3} \mathrm{H}$ thymidine incorporation into DNA. B-lactoglobulin variants $(\square$, variant $A ; \bullet$, variant $B ; \boldsymbol{E}$, variant $A / B$ ) were tested in the absence of fetal calf serum (FCS) (A) or in the presence of $1 \%$ FCS (B). Each result represents the average of 6 replicates.

L'optimum d'activité est obtenu pour une concentration de $3 \mathrm{~g} / \mathrm{l}$ pour les variants $\mathrm{A}$ et $A / B$, et pour une concentration de 0,75 $\mathrm{g} / \mathrm{l}$ pour le variant $\mathrm{B}$. L'activité maximale reste toujours inférieure à celle obtenue en présence de $10 \%$ de SVF pour les variants $A, A / B, B$. Le variant $A$ est significativement plus actif que le variant $B(P<0,05$, test ANOVA). En présence de $1 \%$ de SVF, l'activité mitogénique maximale augmente, elle atteint 71,70 , et $58 \%$ de celle du sérum de veau à $10 \%$ respectivement pour les variants $A, A / B, B$.

L'activité du lactosérum (20 g/l de protéines) est égale à $72 \%$ de celle du SVF, équivalente à celle des variants $A$ et $A / B$.

\section{Test de prolifération cellulaire}

Ces expériences ont été réalisées sur 2 souches d'hybridomes (Mark 3 et antiHPL). Le milieu de culture de base (RPMI 1640) a été supplémenté soit avec $10 \%$ de
SVF (témoin positif), soit avec $9 \%$ de lactosérum et $1 \%$ de SVF, soit $2,7 \mathrm{~g} / \mathrm{l}$ de $\mathrm{B}-\mathrm{Lg}$ variant $A / B$ et $1 \%$ de SVF; cette concentration de B-Lg correspond aux taux physiologiques de la $B-L g$ dans le lait bovin et à l'optimum d'activité déterminés préalablement. A chaque passage est mesuré le nombre de cellules vivantes, de cellules mortes (figs 3 et 4). Dans le milieu de culture est dosé le taux d'immunoglobulines sécrétées (tableau I). II n'y a pas de différence significative (test ANOVA) entre la prolifération cellulaire dans les milieux supplémentés en lactosérum et en B-Lg. Dans les 2 cas, la prolifération cellulaire représente environ $75 \%$ de celle obtenue dans le milieu supplémenté avec $10 \%$ de SVF. II est à noter que le nombre des cellules mortes est plus faible dans les milieux supplémentés en lactosérum et $\beta$-Lg que dans celui supplémenté en SVF. II n'y a pas de différence significative, dans la sécrétion des immunoglobulines, quel que soit le milieu utilisé. 


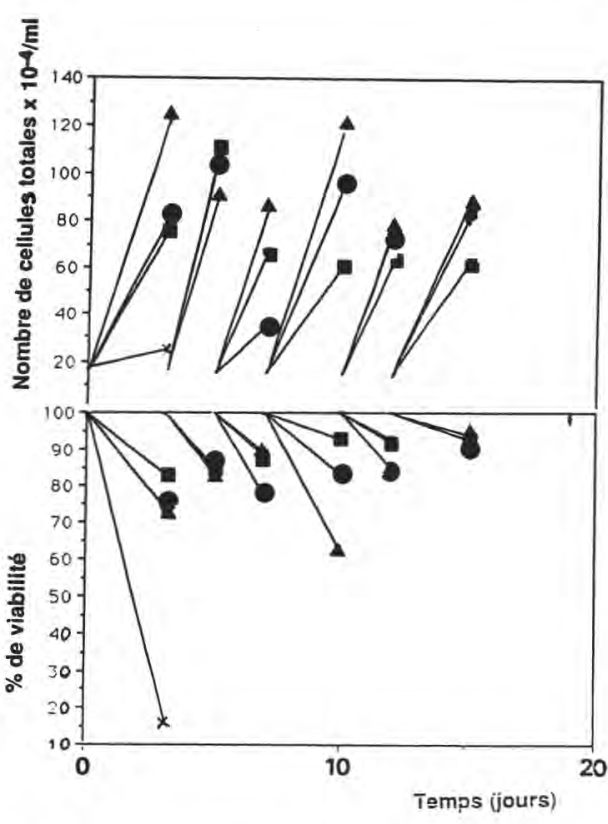

Fig 3. Prolifération de l'hybridome Mark 3 dans différents milieux de culture. $\boldsymbol{\Delta}$ : milieu supplémenté avec $10 \%$ de SVF; : avec $2,7 \mathrm{~g} / \mathrm{l}$ variant $A / B$ de la B-lactoglobuline $+1 \%$ de SVF; - : avec $9 \%$ de lactosérum ( $20 \mathrm{~g} / \mathrm{l}$ de protéines) $+1 \%$ de SVF; X : avec $1 \%$ de SVF. A. Nombre total des cellules; B. Pourcentage des cellules vivantes. Les cellules sont ré-ensemencées tous les 2 ou 3 jours à la concentration de départ, c'est-à-dire $15 \times 10^{4}$ cellules vivantes $/ \mathrm{ml}$. Les résultats sont la moyenne de 3 flacons de $25 \mathrm{~cm}^{2}$ et de 3 comptages par flacon.

Mark 3 hybridoma proliferation in different culture media. $\mathbf{\Delta}$ : medium supplemented with $10 \%$ FCS; : supplemented with $2.7 \mathrm{~g} / \mathrm{l}$ of $\mathrm{B}$ lactoglobulin $A V B+1 \%$ FCS; : supplemented with $9 \%$ whey + $1 \%$ FCS; $X$ : supplemented with $1 \%$ FCS. A. Percentage of living cells. B. Number of dead cells. The cells were seeded again each for 2 or 3 days at the same initial concentration (15 × $10^{4}$ living cells $\left./ \mathrm{ml}\right)$. The results were the average of $3 \times 25 \mathrm{~cm}^{2}$ flasks and 3 counts per flask.

\section{Rôles respectifs du sérum de veau et de la $\beta$-lactoglobuline}

Les expériences précédentes de prolifération cellulaire ont été menées en mainte-

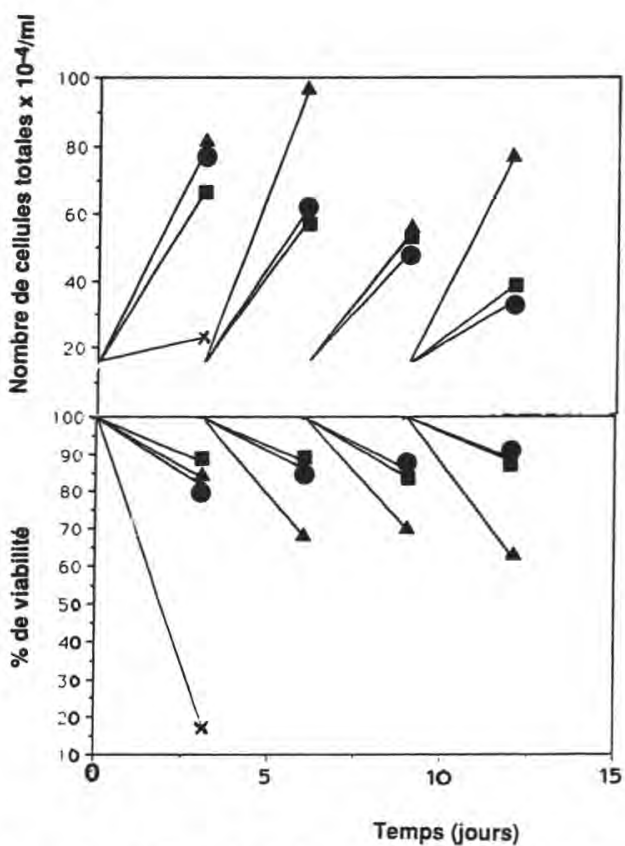

Fig 4. Prolifération de l'hybridome anti-HPL dans différents milieux de culture. $\mathbf{\Delta}$ : milieu supplémenté avec $10 \%$ de SVF; : avec $2,7 \mathrm{~g} / \mathrm{I}$ variants $A / B$ de la B-lactoglobuline $+1 \%$ SVF; - : avec $9 \%$ de lactosérum (protéines $20 \mathrm{~g} / \mathrm{l}$ ) + $1 \%$ SVF; X : avec $1 \%$ de SVF. A. Nombre de cellules vivantes; B. Pourcentage des cellules vivantes. Les cellules sont ré-ensemencées tous les 2 ou 3 jours à la concentration de départ, c'est-à-dire $15 \times 10^{4}$ cellules vivantes $/ \mathrm{ml}$. Les résultats sont la moyenne de 3 flacons de $25 \mathrm{~cm}^{2}$ et de 3 comptages par flacon.

Anti-HPL hybridoma proliferation in different culture media. A: medium supplemented with $10 \%$ FCS; : supplemented with $2.7 \mathrm{~g} / \mathrm{l}$ of $\beta$ lactoglobulin AVB + 1\% FCS; $\bullet$ : supplemented with $9 \%$ whey $+1 \%$ FCS; $X$ : supplemented with $1 \%$ FCS. A. Total number of cells. B. Percentage of living cells. The cells were seeded each for 2 or 3 days at the same initial concentration $\left(15 \times 10^{4}\right.$ living cells $\left./ \mathrm{m} /\right)$. The results were the average of $3 \times 25 \mathrm{~cm}^{2}$ flasks and 3 counts per flask.

nant $1 \%$ de SVF dans le milieu supplémenté avec la B-Lg. II nous a paru intéressant de déterminer les rôles respectifs de la $\beta-\mathrm{Lg}$ et du SVF dans la prolifération cellulaire. Pour cela, deux séries d'expériences ont été réalisées. 
Tableau I. Sécrétion d'anticorps monoclonaux en fonction du milieu de culture (mg/l). I) RPMI 1640 supplémenté avec $10 \%$ de sérum de veau fœtal; II) lactosérum $9 \%(20 \mathrm{~g} / \mathrm{l}$ de protéines de lait) $+1 \%$ de SVF; III) B-lactoglobuline variant A/B $(2,7 \mathrm{~g} / \mathrm{l})+1 \%$ de SVF. Les résultats sont la moyenne obtenue sur les surnageants de 3 boîtes de $25 \mathrm{~cm}^{2}$ testés chacun 3 fois. L'écart type est faible, toujours inférieur à $\pm 0,01 \mathrm{mg} / \mathrm{l}$.

Monoclonal antibody secretion according to culture medium composition (mg/l). I) RPMI 1640 supplemented with $10 \%$ fetal calf serum (FCS). II) Whey $9 \%$ (20 g/l milk proteins) $+1 \%$ FCS. III) Variant AVB of $\beta$-lactoglobulin $(2.7 \mathrm{~g} / \mathrm{l})+1 \%$ FCS. Results are the mean from supernatant of 3 culture boxes (25 $\mathrm{cm}^{2}$ ), each tested 3 times. The standard deviation is always less than $\pm 0.01 \mathrm{mg} /$.

Temps de culture

(j)
Hybridome Mark 3

Milieux
Hybridome anti-HPLC Milieux

$\begin{array}{lllll}\text { I } & \text { II } & \text { III } & \text { I } & \text { II }\end{array}$

\begin{tabular}{rcccccc}
\hline 3 & 15,1 & 15 & 15 & 10,5 & 11,1 & 10,9 \\
5 & 13 & 19,1 & 16 & - & - & - \\
6 & - & - & - & 11,5 & 10,8 & 10,2 \\
7 & 16,2 & 10,7 & 15,5 & - & - & - \\
9 & 17,3 & 19,7 & 17,1 & 10,5 & 9,7 & 11,8 \\
11 & 15,1 & 15,1 & 15,1 & - & - & - \\
12 & - & - & - & 11,9 & 10,3 & 10 \\
15 & 13,1 & 15,1 & 15,1 & - & - & - \\
\hline
\end{tabular}

Dans la première, on abaisse progressivement la concentration de SVF dans le milieu de culture de 10 à $1 \%$. Les cultures sont maintenues 9-12 j, sauf pour la concentration de $1 \%$ de SVF où les cellules meurent au bout de 2-3 j. La figure 5 donne le nombre de cellules vivantes, comptées au $3^{e} \mathrm{j}$ de culture, en fonction de la concentration en SVF. La prolifération cellulaire diminue progressivement, parallèlement à l'appauvrissement du milieu en SVF. Elle représente environ 63 et $38 \%$ de celle obtenue avec $10 \%$ de SVF, pour une concentration en SVF de 3 et $2 \%$ respectivement; une concentration à $1 \%$ de SVF ne peut assurer seule la croissance cellulaire à long terme.

Dans la seconde série d'expériences, 2,7 $\mathrm{g} / \mathrm{l}$ de $\beta$ - $\mathrm{Lg}$ variant $\mathrm{A} / \mathrm{B}$ sont maintenus dans le milieu de culture et la concentration de SVF est diminuée de 1 à $0 \%$. On

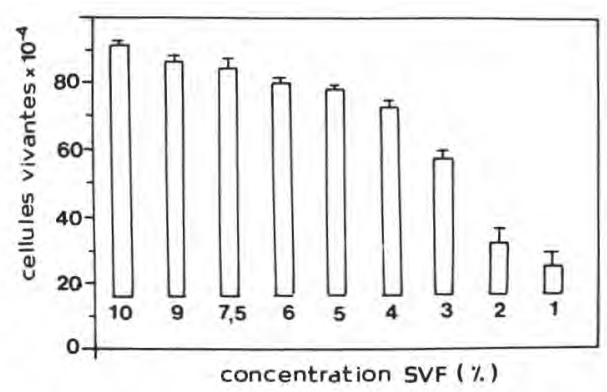

Fig 5. Prolifération de l'hybridome Mark 3 en fonction de la concentration en SVF dans le milieu de culture. Les cellules sont comptées au $3^{\mathrm{e}} \mathrm{j}$ de culture. Les résultats sont la moyenne des densités cellulaires de 3 flacons de $25 \mathrm{~cm}^{2}$ et de 3 comptages par flacon.

Mark 3 hybridoma proliferation according to FCS concentration in the culture medium. The cells were counted on the third day of culture. The results were the average cell densities of 3 $x 25 \mathrm{~cm}^{2}$ and 3 counts per flask. 
constate (fig 6) que la $\beta$ - $\mathrm{Lg}$ seule est insuffisante pour permettre la prolifération à long terme. L'abaissement de la concentration en SVF entraîne une diminution significative de la croissance celiulaire $(P<0,05$, test ANOVA) et de la sécrétion des immunoglobulines (tableau II).

\section{DISCUSSION}

Dans ce travail, nous avons vérifié si la BLg pouvait être utilisée comme agent mitogène dans les milieux de culture, en remplacement du lactosérum qui reste un milieu complexe. Cette protéine n'a pas été utilisée à notre connaissance pour la supplémentation des milieux de culture.

Des tests de croissance à court terme (synthèse d'ADN) révèlent des différences dans l'activité mitogénique des variants $A$ et $B$, qui peuvent s'expliquer au moins en

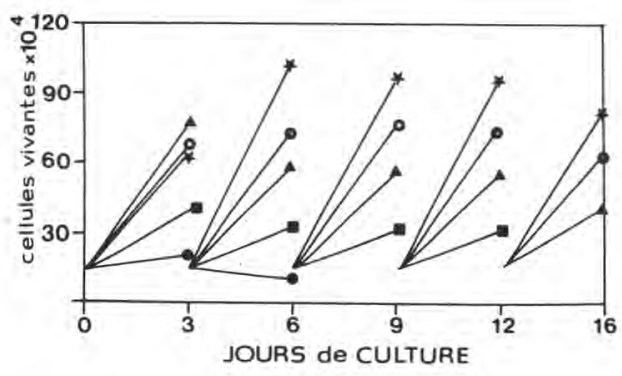

Fig 6. Prolifération de l'hybridome Mark 3 dans des milieux de culture supplémentés avec 2,7 g/l de B-lactoglobuline variant $A / B$ et des concentrations décroissantes de SVF. $\star$ : $1 \%$; $0: 0,75 \% ; \mathbf{\Delta}: 0,5 \%$; $: 0,25 \%$; $0 \%$. Les résultats sont la moyenne des densités cellulaires de 3 flacons de $25 \mathrm{~cm}^{2}$ et de 3 comptages par flacon.

Mark 3 hybridoma proliferation in culture medium supplemented with $2.7 \mathrm{~g} / \mathrm{l}$ of $\mathrm{B}$ lactoglobulin $A / B$ and increasing FCS concentrations. $\star: 1 \% ; 0: 0.75 \% ; \mathbf{\Delta}: 0.5 \% ; \boldsymbol{\nabla}: 0.25 \%$; - $0 \%$. The results were the average cell densities of $3 \times 25 \mathrm{~cm}^{2}$ flask with 3 counts per flask. partie par des propriétés physicochimiques différentes:

- la solubilité : le variant $A$ est plus soluble (3 g/l) que le variant B $(0,6 \mathrm{~g} / \mathrm{l})$ (Alais, 1984);

- modification de la conformation en fonction du $\mathrm{pH}$ du milieu : au-dessus de $\mathrm{pH} 6,8$ (les milieux de culture sont tamponnés à $\mathrm{pH} 7,2$ ), la molécule subit une transition particulière (Pessen et al, 1985), qui entraîne le démasquage d'un groupement carboxylique et la réactivité du groupement SH 121 (Georges et al, 1962; Timasheff et al, 1966; Mc Kenzie et Sawyer, 1967; Tandford, 1968 et 1970; McKenzie, 1971);

Tableau II. Sécrétion d'anticorps monoclonaux (hybridome Mark 3; mg/l) en fonction de la concentration en sérum de veau fœtal. Tous les milieux contiennent de la $\beta$-lactoglobuline variant $A / B$ 2,7 $\mathrm{g} / 1$ supplémentée avec $1 \%$ SVF (A), $0,75 \%$ SVF (B), $0,5 \%$ SVF (C), $0,25 \%$ SVF (D), $0 \%$ SVF $(E)$. Les résultats sont la moyenne obtenue sur les surnageants de 3 boîtes de 25 $\mathrm{cm}^{2}$ testés chacun 3 fois. L'écart type de ces mesures est faible, toujours inférieur à $\pm 0,01$ $\mathrm{mg} / \mathrm{l}$.

Monoclonal antibody secretion (mg/l) according to FCS concentration in culture medium. All the media contain $2.7 \mathrm{~g} / \mathrm{l}$ of $\beta$-lactoglobulin variant AVB supplemented with $1 \%$ FCS (A), $0.75 \%$ FCS (B), 0.5\% FCS (C), 0.25\% FCS (D), 0\% FCS (E). Results are the means obtained from supernatant of 3 culture boxes $\left(25 \mathrm{~cm}^{2}\right)$, each tested 3 times. The standard deviation is always less than $\pm 0.01 \mathrm{mg} /$.

\begin{tabular}{rrrrrr}
\hline $\begin{array}{c}\text { Temps } \\
\text { de culture } \\
\text { (j) }\end{array}$ & A & B & $C$ & $D$ & $E$ \\
& & & & & \\
\hline 3 & 15,4 & 7,4 & 15,5 & 7,4 & 2,4 \\
6 & 15,3 & 15,3 & 10,4 & 2,5 & 0,25 \\
9 & 15,8 & 7,2 & 0,7 & - & - \\
12 & 15,8 & 7,2 & 0,7 & - & - \\
15 & 11,4 & 7 & - & - & - \\
\hline
\end{tabular}


ceci impliquerait un effet de charge plus important sur le variant $A$, en raison d'un résidu aspartyl en position 64 , alors que le variant $\mathrm{B}$ a un résidu glycyl à cette position (Eigel et al, 1984);

- I'hydrophobicité : le variant $A$ est un peu plus hydrophobe que le variant $B$ (Cheftel et al, 1985); cependant, l'exposition à la surface de la molécule du variant $A$ du résidu aspartyl 64 (Monaco et al, 1987) pourrait réduire la fixation de composés sur les zones hydrophobes (lle 56, Leu 57 et 58, Trp 61, lle 147, Leu 149 et Phe 151).

Lorsqu'on étudie la prolifération cellulaire, on constate que les milieux supplémentés avec 2,7 g/l de B-Lg variant A/B et $1 \%$ de SVF sont aussi efficaces que les milieux supplémentés avec $9 \%$ de lactosérum. Donc, l'activité mitogénique du lactosérum semble être liée, au moins pour la plus grande partie, à la présence de la $\beta$ $\mathrm{Lg}$. La $\beta-\mathrm{Lg}$, outre son rôle de nutriment, est un transporteur de substances hydrophobes et de métaux (Baumy et Brulé, 1988), ce qui peut expliquer son effet mitogène. Par ailleurs, il y a moins de cellules mortes dans les cultures supplémentées avec les produits provenant du lait qu'avec du SVF. La $\beta$-Lg aurait un effet protecteur, effet déjà rapporté par Polet et PoletSpieker (1976) qui l'utilisent associée à la concanavaline $A$, dans des cultures de lymphocytes à court terme, et que nous avions également mis en évidence en étudiant la congélation des cellules dans du lactosérum (Derouiche et al, 1989).

Par contre, nous constatons que ni la BLg seule $(2,7 \mathrm{~g} / \mathrm{l})$, ni le SVF seul à $1 \%$ ne sont capables d'assurer la prolifération cellulaire à long terme, alors qu'ajoutés ensemble, ils agissent en synergie pour assurer une croissance cellulaire qui est d'environ $75 \%$ de celle obtenue avec $10 \%$ de SVF. La concentration de $1 \%$ de SVF semble être la concentration minimale requise pour assurer en synergie avec la B-
Lg une prolifération cellulaire et une sécrétion correcte d'anticorps.

La technique utilisée pour la préparation de la B-Lg est simple, peu coûteuse, elle donne un rendement et un degré de pureté corrects, ce qui fait qu'elle est facilement utilisable pour la préparation de milieux de culture à grande échelle.

\section{REMERCIEMENTS}

Nous remercions l'équipe "protéinologie" du laboratoire de biochimie appliquée à l'université de Nancy I pour l'aide technique lors de la réalisation de la chromatographie en FPLC.

Ce travail a pu être réalisé grâce à l'aide financière de l'université de Nancy I, du district de Nancy, de la mission de la recherche de la région Lorraine, et grâce à un contrat $\mathrm{CEE}$ (contrat BAP 0/0129 F).

\section{RÉFÉRENCES}

Alais C (1984) Science du lait : principes de techniques laitières. Sepaic, Paris, $4^{e}$ édn

Baumy JJ, Brulé G (1988) Binding of bivalent cations to $\alpha$-lactalbumin and $\beta$-lactoglobulin: effect of $\mathrm{pH}$ and ionic strength. Lait $68,33-48$

Brown ME (1984) Interactions of $\beta$-lactoglobulin and $\alpha$-lactalbumin with lipids: a review. J Dairy Sci 67, 713-722

Cheftel JC, Cuq JL, Lorient D (1985) Protéines alimentaires. Propriétés fonctionnelles. Valeur nutritionnelle. Modifications chimiques. Technique et documentation Lavoisier, Paris

Damerdji O, Derouiche F, Legrand C, Capiaumont J, Bour JM, Maugras M, Belleville F, Nabet $P$, Paquet D, Linden G (1988) Utilisation of whey fractions as substitute for fetal calf serum in culture media. Biotechnol Tech 2, 253-258

Derouiche F, Bour JM, Legrand C, Capiaumont J, Belleville F, Linden G, Nabet P (1989) Improved long-term storage of hybridomas at $80^{\circ} \mathrm{C}$ using a bovine milk derivative. J Immunol Methods 125, 13-18 
Derouiche AF, Legrand C, Bour JM, Capiaumont J, Gelot M, Dousset B, Belleville F, Nabet $P$, Linden $G(1990)$ Biochemical aspects of whey fractions capable of monitoring hybridome proliferation. Comparison with fetal calf serum. Lait 70, 313-324

Diaz de Villegas MC, Oria R, Sala FJ, Calvo $M$ (1987) Lipid binding by $\beta$-lactoglobulin of cow milk. Milchwissenschaft 42, 357-358

Douillard JY, Hoffman T (1983) Enzyme-linked immuns sorbant assay for screening monoclonal antibody production using enzymelabeled second antibody. Methods Enzymol 92, 168-175

Eigel WN, Butler JE, Ernstrom CA, Farrel HMJr, Harwalkar VR, Jenness R, Whitney RM (1984) Nomenclature of proteins of cow's milk. Fifth revision. J Dairy Sci 67, 16111613

Fugate RD, Song PS (1980) Spectroscopic characterization of $\beta$-lactoglobuline-retinol complex. Biochim Biophys Acta 625, 28-42

Georges C, Guinand S, Tonnelat J (1962) Étude thermodynamique de la dissociation réversible de la $\beta$-lactoglobuline $B$ pour des $\mathrm{pH}$ supérieurs à 5,5. Biochim Biophys Acta 59, 737-739

Godovac-Zimmermann J (1988) The structural motif of $\beta$-lactoglobulin and retinol-binding protein: a basic framework for binding and transport of small hydrophobic molecules? Trends Biochem Sci 13, 64-67

Godovac-Zimmermann J, Braunitzer G (1987) Modern aspects of the primary structure and function of $\beta$-lactoglobulins. Milchwissenschaft 42, 294-297

Godovac-Zimmermann J, Conti A, Liberatori J, Braunitzer G (1985) Homology between the primary structure of $\beta$-lactoglobulins and human retinol binding protein: evidence for a similar biological function? Biol Chem Hoppe-Seyler 366, 431-438

Hillier RM (1976) The quantitative measurement of whey proteins using polyacrylamide - gel electrophoresis. J Dairy Res 43, 259-261

Klagsbrun M (1978) Human milk stimulates synthesis and cellular proliferation in cultured fibroblasts. Proc Natl Acad Sci (USA) 75, 5057-5061

Linden G, Nabet P, Bour JM (1988) Compléments de milieux de culture de cellules eucaryotes à base de produits dérivés de l'industrie laitière. Brevet français $n^{\circ} 8815723$
Lung C, Paquet D, Linden G (1987) Isolement de la $\beta$-lactoglobuline. Mise au point d'une méthode préparative rapide. Sci Alim $7\left(n^{\circ}\right.$ hors série VIII), 167-176

McKenzie HA (1971) $\beta$-lactoglobulins. In: Milk proteins; chemistry and molecular biology (McKenzie HA, ed) 2. Acad Press, New York, 257-330

McKenzie HA, Sawyer WH (1967) Effects of pH on $\beta$-lactoglobulins. Nature 214, 1101-1104

Monaco HL, Zanotti G, Spadon P, Bolognesi M, Sawyer L, Eliopoulos EE (1987) Crystal structure of the trigonal form of bovine $\beta$ lactoglobulin and its complex with retinol at $2.5 \AA$ resolution. J Mol Biol 197, 695-706

Nabet P, Linden G, Ledeaut Y, Sarem F (1985) Milieu de culture à base de lait de vache. Brevet Anvar, France, 8512907

Papiz MZ, Sawyer L, Eliopoulos EE, North ACT, Findlay JBC, Sivaprasadarou $R$, Jones TA, Newcomar ME, Kraulis PJ (1986) The structure of $\beta$-lactoglobulin and its similarity to plasma retinol-binding protein. Nature 324, 383-385

Pessen H, Purcell JM, Farrell HMJr (1985) Proton relaxation rates of water in dilute solutions of $\beta$-lactoglobulin. Determination of cross relaxation and correlation with structural changes by the use of two genetic variants of a self-associating globular protein. Biochim Biophys Acta 828, 1-12

Polet H, Polet-Spieker H (1976) Mechanism of the growth-promoting effect of serum albumin on concanavalin A actived lymphocytes: protective effect of the plasma proteins. $J$ Immunol 117, 1275-1281

Polet-Spieker H, Polet H (1981) Requirement of a combination of a saturated and unsaturated free fatty acid and a fatty acid carrier protein for in vitro growth of lymphocytes. J Immunol $126,949-954$

Rask L, Anundi H, Peterson PA (1979) The primary structure of the human retinol-binding protein. FEBS Lett 104, 55-58

Said HM, Ong DE, Shingleton JL (1989) Intestinal uptake of retinol: enhancement by bovine milk $\beta$-lactoglobulin. Am J Clin Nutr 49, 690694

Sereni A, Baserga R (1981) Routine growth of cell lines in medium supplemented with milk instead of serum. Cell Biol Int Rep 5, 339345 
Tandford C (1968) Protein denaturation. In: Advances in protein chemistry - 23 (Auginsen CBJr, Anson ML, Edsall JT, Richards FM, eds) Acad Press, New York, 121-282

Tandford C (1970) Protein denaturation. In: Advances in protein chemistry - 24 (Auginsen
CBJr, Edsall JT, Richards FM, eds) Acad Press, New York, 1-95

Timasheff SN, Mescanti L, Basch JJ, Townend $R$ (1966) Conformational transitions of bovine $\beta$-lactoglobulins A, B and C. J Biol Chem $241,2496-2501$ 